\title{
Fuzzy set theory applied to bend sequencing for sheet metall bending
}

\author{
S.K. Ong ${ }^{\mathrm{a}, *}$, L.J. De Vin ${ }^{\mathrm{b}}$, A.Y.C. Nee ${ }^{\mathrm{a}}$, H.J.J. Kals ${ }^{\mathrm{c}}$ \\ a Mechanical \& Production Engineering Department, National University of Singapore, 10 Kent Ridge Crescent, Singapore 119260, Singapore \\ b Advanced Forming Tech. Group, University of Ulster at Jordanstown, Jordanstown, UK \\ ${ }^{c}$ Mechanical Engineering Department, University of Twente, Netherlands
}

Received 15 December 1995

\begin{abstract}
Brake forming is widely applied in the high variety and small batch part manufacturing of sheet metal components, for the bending of straight bending lines. Currently, the planning of the bending sequences is a task that has to be performed manually, involving many heuristic criteria. However, set-up and bend sequencing procedures and knowledge have to be formally formalized and modeled, for the development of computer-aided process planning systems for sheet metal forming. This paper describes the application of fuzzy set theory for the normalization and modeling of the set-up and bend sequencing process for sheet metal bending. A fuzzy-set based methodology is used to determine the optimal bending sequences for the brake forming of sheet metal components, taking into account the relative importance of handling and accuracy. (C) 1997 Elsevier Science S.A.
\end{abstract}

Keywords: Sheet metal; Fuzzy set theory; Bending

\section{Introduction}

In small batch and high variety sheet metal part manufacturing, bends are usually made by brake forming or swing-type bending. As a result, each bend involves a separate set-up. Therefore, the number of set-ups can be relatively large in bending. The fact that the shape of a part changes both locally and globally presents some additional problems, one of which being that tolerance reasoning in set-up determination for sheet metal parts is more complicated than that for the case of prismatic or rotational parts. A formal description of the bend sequencing procedures is also more difficult. However, a formal description of the sequencing procedures is required when these procedures are to be incorporated in computer-aided process planning (CAPP) systems.

In set-up sequencing for sheet metal bending, five sub-functions can be distinguished [1]. These five sub-

\footnotetext{
* Corresponding author. Fax: +65 7791459; e-mail: mpeongsk@nus.sg
}

functions are: (i) a reduction of the tolerance scheme; (ii) a reduction of the search space; (iii) the generation of alternative candidate set-ups; (iv) a selection from the set of candidate set-ups; and (v) a collision check. These five sub-functions must be performed in both manual and computer-aided set-up determinations. This paper focuses on the handling and collision-checking aspects of set-up determination for brake forming, the accuracy aspect being described elsewhere [1].

An important aspect of solving a problem is the modelling and representation of the knowledge and data of the problem. The representation technique chosen should appropriately capture and represent the knowledge and data without causing omission and inconsistency. In sheet metal set-up sequencing and bending, heuristic rules are often employed [1,2]. These rules-of-thumb entail some level of intuition and fuzziness. The theory of fuzzy set originally developed by Zadeh [3] provides an adequate conceptual framework as well as a mathematical tool to solve real world problems that are usually fuzzy. Many researchers have reported the use of fuzzy set theory in areas such as 

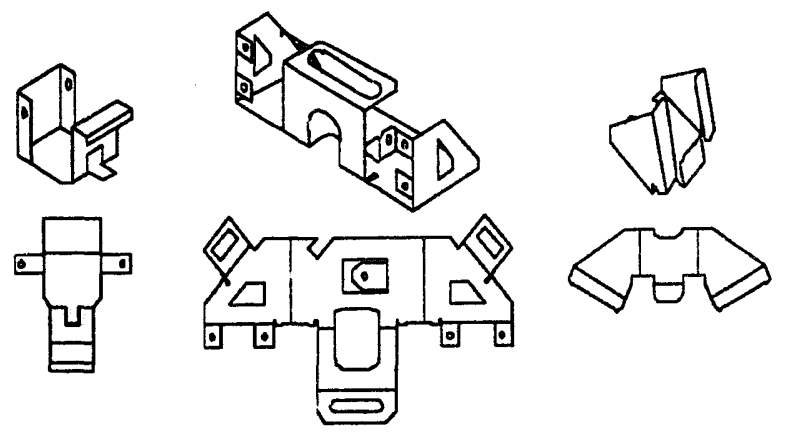

Fig. 1. Examples of sheet metal components.

sheet bend design [4], machining set-up planning [5], and three-roll bending [6]. In the present study, the optimum bend and set-up sequence in sheet metal forming is determined by combining the tolerance analysis with fuzzy reasuning. This combination allows the relative importance of the handling and the accuracy criteria to be considered in the sequencing process.

\section{Sheet metal part manufacturing: Brake forming}

Order-based small batch part manufacturing is a high-variety, low-volume production environment. In sheet metal part manufacturing, customer demands have led to decreasing batch sizes and increasing accuracy demands. Fig. 1 shows a few examples of sheet metal components. In small batch part manufacturing, bends are straight and made on bending machines employing swing-type bending processes or brake forming. The tools used in these processes are general purpose tools, as opposed to forming processes such as deep drawing (automotive industry) and rubber pad forming (aerospace industry), where dedicated tools are used for each component type. Swing-type bending is mainly used for components such as panels and sashes, and is less suited for use in a high variety environment.

Fig. 2 shows a set-up for brake forming. The sheet or the partly bent component is positioned against two finger stops, and subsequently clamped between the punch and die. Next, the finger stops may be retracted.

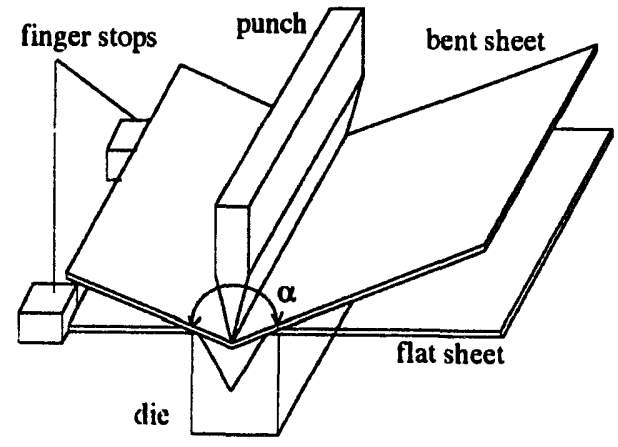

Fig. 2. A set-up for brake forming.
In many cases, however, they can stay in position. The punch is then lowered to its final position and retracted.

Sheet metal part manufacturing has followed developments found earlier in machining [1,7]. It has developed rapidly into flexible manufacturing with the use of CNC equipment and it is turning out high-value-added components. Equipment found nowadays in sheet metal manufacturing includes $\mathrm{CNC}$ punch presses for processing sheets of up to $1.5 \times 4 \mathrm{~m}$ with several different components nested in one sheet, laser cutting machines, laser presses and $\mathrm{CNC}$ press brakes [8]. Increased component variety and accuracy demands, and shorter delivery times, have increased the workload in process planning. Process planning tasks such as tool path calculations for punching and nibbling are becoming increasingly automated [1]. In many cases, however, set-up planning is still being performed manually [9]. Automated systems for set-up planning for brake forming are almost without exception variant or interactive type systems $[2,10-12]$.

\section{Set-up planning in brake forming}

In the conventional procedures, a process planner examines the workpiece model and applies a particular strategy, which can vary from case to case, to find the bending sequence. For example, when a component has a complex structure but moderate tolerance prescriptions, the process planner will first try to find a collision-free bending sequence and pay attention to the accuracy aspect later. The way in which collision checking is addressed may also depend on the complexity of the component. In finding a bending sequence, the process planner moves back and forth through the five sub-functions mentioned in Section 1.

In a CAPP system, the sub-functions are clearly separated and executed in a given sequence. Nevertheless, the procedures and strategies in conventional planning find their counterparts in the procedures in CAPP. A CAPP system is able to investigate a large number of alternatives thoroughly. However, the search space can be very large and must therefore be restricted by identifying non-feasible solution paths quickly. In addition, pruning of alternatives is required after each process step to avoid explosion of the number of alternatives.

The collision aspect can be addressed with collision checking or collision prediction. Many iterations may be required when only a check is used. On the other hand, the prediction of collisions with the use of algorithms can be a problem. The algorithms must be capable of identifying all of the restrictions without excluding feasible alternatives. Therefore, a hybrid method is often the best solution.

A 'forward' or a 'reverse' bending sequence can be generated. A forward bending sequence has the flat 
pattern as a starting point, whilst the reverse bending sequence starts with the completed component and the bends are unfolded until the flat pattern stage is reached. The reverse bending sequence has the advantage that restrictions in the bending sequence are identified more readily. The advantage of knowing the 'bending history' of a component in forward bending is limited, as the influence of imperfections on the overall accuracy remains uncertain. The accuracy aspect can be addressed adequately with the use of tolerance trees for bending when a reverse bending sequence is generated [1]. In this paper, the reverse bending sequence method is used.

When the number of part configurations is limited, a classification can aid in the generation of bending sequences [13]. In a small-batch and high-variety environment, this method is limited in its application although different strategies can be used for different component types $[9,14]$. In this paper, a step-wise generative method is used. In a given intermediate state, candidate preceding states are generated and the most promising alternative is selecied to proceed with during the next process step. This technique is called 'local ordered depth first search'. It is very suitable when an optimal solution is not required, but a feasible solution must be found, which is the case in sheet metal set-up planning.

\section{Set-up sequencing rules}

\subsection{Selection of a central face}

The definition of a 'central face' is very useful for distinguishing different types of bends that have to be made, and for describing the rotations of a part between different set-ups. The selection of a central face from the main faces present in the component must be based on meaningful criteria. The way in which a face is surrounded by other faces is important for identifying a 'central face'. When the surrounding faces are equally balanced over the bends attached to a face, the latter face is situated 'in the middle' of the product structure. The size of a face is the second criterion used when the first criterion does not result in the unambiguous selection of a central face. The definition of the 'variance' from the probability calculus can be adopted for determining the 'central face' $[1,15]$. Faces on the periphery of the product structure, i.e., faces that are connected to the part with only one bend, are called 'single faces'. In Fig. 3, faces B, C, D and F are single faces whilst face $A$ is the central face.

\subsection{Handling rules and criteria}

At each stuge, the set-up planning procedure involves selecting a bend to unfold, based on the bend that has

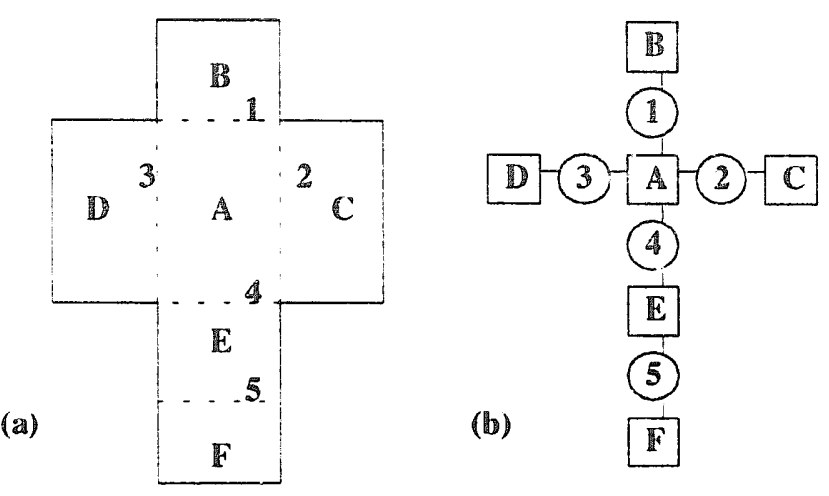

Fig. 3. Sample Part: (a) flat pattern; and (b) product structure.

been currently unfolded. In this section, the handling rules and criteria used for determining the unfolding bend sequence are discussed. These rules deal with the selection of the next bend for unfolding. Each of these rules establishes relationships between pairs of bends, concerning a particular criterion. The degrees of satisfaction of the criteria when particular bends are selected as the next bend to be unfolded, are not equal for all bends. A high membership grade indicated for a particular rule means that the bend is a good selection to unfold next, according to the rule. These relationships between the bends and criteria are represented as fuzzy relations, and the membership grades of these fuzzy relations are determined through fuzzy membership functions, as shown in Fig. 4.

\subsubsection{Compound bend rule}

Compound bends are bends with the same bend angie and bend radius, and a common bend axis. In addition, the distance between the two lines through the bend axes is zero. Compound bends are preferably bent in one set-up, especially when they do not have a common adjacent face. Processing another bend first can 'break' the common bend axis into two different axes. After this, the bends can no longer be processed in one set-up. For this rule, the membership value for the relation between a pair of bends would be either unity when the pair forms a compound bend, and zero otherwise.

\subsubsection{Parallel bends rule}

This rule is formulated as follows: "after a bend is bent, proceed with the nearest parallel bend, when this bend is situated on the same side of the central face." 'On the same side' means that there is a connection from the first bend to the other bend in the product structure that does not contain the central face. The membership function in Fig. 4(a) for this rule is formulated such that if there are fewer bends between the bend that has been currently unfolded and the bend that is being considered for unfolding next, the relation- 
(a) Parallel Bend Rule

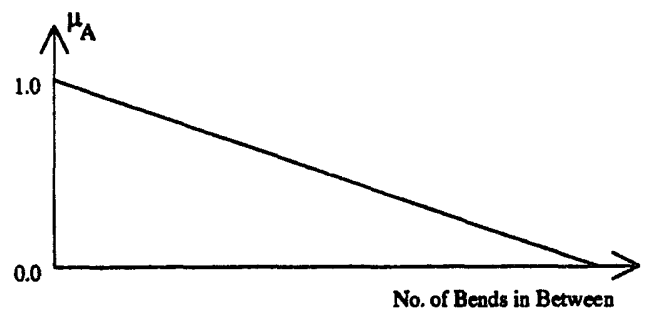

(c) Single Face Rule

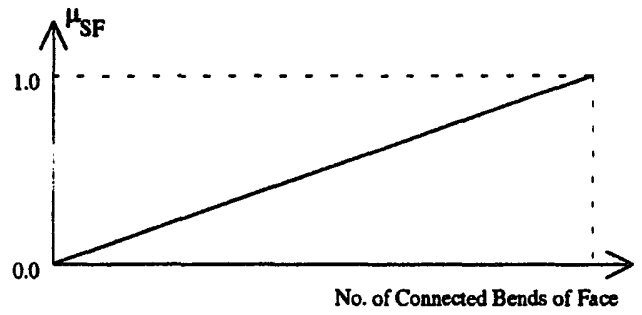

(b) Shape Determining Bend Rule

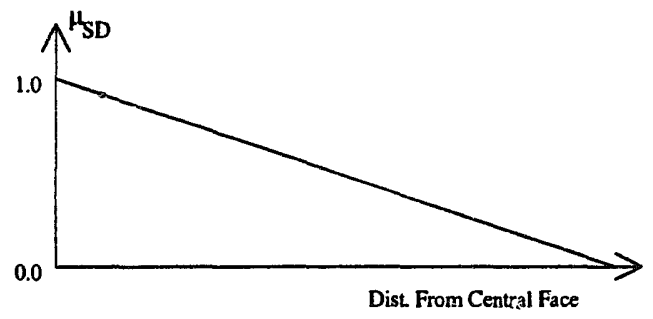

(d) Combined Rotation Rule

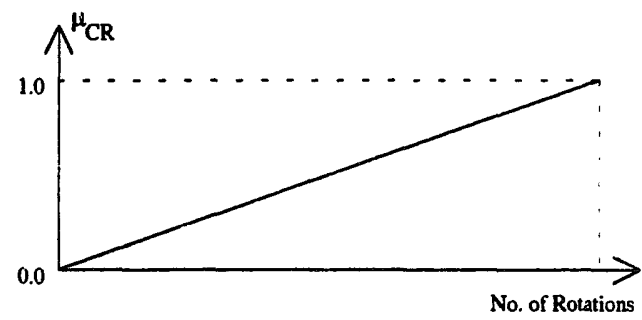

Fig. 4. Fuzzy variables in the handling rules.

ships based on this criterion would have lower values than when there are more bends, on the sarne side of the central face.

\subsubsection{Shape determining bend rule}

A shape-determining bend is a bend next to the central face. Usually, the other adjacent face of the bend is also relatively large. After a 'shape-determining bend' has been bent, it becomes more difficult to position the part on the press brake, and to access the other bends with the punch. Therefore, a fair strategy is to bend the 'shape-determining bends' after the other bends. The membership grades of the relationships that are formed as a result of this rule are determined using the function in Fig. 4(b). When a bend is further from the central face, the grade of the relationship will be higher since it would not be a 'shape-determining bend', and thus can be unfolded last or bent first.

\subsubsection{Single face rule}

As mentioned, a single face is connected with only one bend to the part. On the average, this bend has only a minor influence on the overall geometry of the part. Therefore. 'single face' bends can be bent in an early stage, without causing problems for the processing of the other bends. $A$ bend that has fewer bends connected to its adjacent faces would be more preferred to be bent first or unfolded last, as illustrated in Fig. 4(c).

\subsubsection{Combined rotation rule}

Handling operations must be kept as simple as possible. The press brake coordinate system can serve as a reference, and the product movements can be described by the movement of the central face, about these axes.
Rotations along two axes must be avoided, as multiple rotations cost more time and may sometimes lead to errors. A bend that would cause more rotations of the component would be preferred to be bent last or unfolded first, as illustrated in Fig. 4(d). Thus, the relationships that are formed as a result of this rule will have higher degrees, when the bend considered will cause many rotations.

\subsection{Accuracy and positioning rules and criteria}

These rules are used to select a set-up for performing a bend from a set of alternative set-ups.

\subsubsection{Main part towards opera or rule}

On the average, the positioning of a component on the press brake is easier when a majur portion of the component is situated on the operator's side of the press brake. This orientation also facilities the support of the component during the bending operation. The latter can be neceseary for large components, especially when a relatively small bend is processed. However, there must be a particular threshold value, as there must be some material on the side of the operator in order to be able to handle the part. The membership

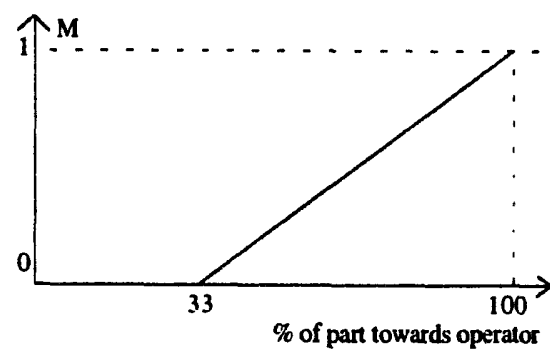

Fig. 5. Main part towards operator rule. 
Table 1

A fuzzy matrix, [S]

\begin{tabular}{llllll}
\hline & $r_{1}$ & $r_{2}$ & $r_{3}$ & $r_{4}$ & $r_{5}$ \\
$b_{1}$ & $\mu_{\mathrm{SD}}\left(b_{1}, r_{1}\right)$ & $\mu_{\mathrm{SF}}\left(b_{1}, r_{2}\right)$ & $\mu_{\mathrm{CR}}\left(b_{1}, r_{3}\right)$ & $\mu_{\mathrm{C}}\left(b_{1}, r_{4}\right)$ & $\mu_{\mathrm{A}}\left(b_{1}, r_{5}\right)$ \\
$b_{2}$ & $\mu_{\mathrm{SD}}\left(b_{2}, r_{1}\right)$ & $\mu_{\mathrm{SF}}\left(b_{2}, r_{2}\right)$ & $\mu_{\mathrm{CR}}\left(b_{2}, r_{3}\right)$ & $\mu_{\mathrm{C}}\left(b_{2}, r_{4}\right)$ & $\mu_{\mathrm{A}}\left(b_{2}, r_{5}\right)$ \\
$b_{3}$ & $\mu_{\mathrm{SD}}\left(b_{3}, r_{1}\right)$ & $\mu_{\mathrm{SF}}\left(b_{3}, r_{2}\right)$ & $\mu_{\mathrm{CR}}\left(b_{3}, r_{3}\right)$ & $\mu_{\mathrm{C}}\left(b_{3}, r_{4}\right)$ & $\mu_{\mathrm{A}}\left(b_{3}, r_{5}\right)$ \\
$b_{4}$ & $\mu_{\mathrm{SD}}\left(b_{4}, r_{1}\right)$ & $\mu_{\mathrm{SF}}\left(b_{4}, r_{2}\right)$ & $\mu_{\mathrm{CR}}\left(b_{4}, r_{3}\right)$ & $\mu_{\mathrm{C}}\left(b_{4}, r_{4}\right)$ & $\mu_{\mathrm{A}}\left(b_{4}, r_{5}\right)$ \\
$b_{5}$ & $\mu_{\mathrm{SD}}\left(b_{5}, r_{1}\right)$ & $\mu_{\mathrm{SF}}\left(b_{5}, r_{2}\right)$ & $\mu_{\mathrm{CR}}\left(b_{5}, r_{3}\right)$ & $\mu_{\mathrm{C}}\left(b_{5}, r_{4}\right)$ & $\mu_{\mathrm{A}}\left(b_{5}, r_{5}\right)$ \\
\hline
\end{tabular}

function graph is as shown in Fig. 5, when the threshold value is set at $33 \%$. This means that at least $33 \%$ of the part must be on the side of the operator. The membership value is unity when $100 \%$ of the part is on the side of the operator.

\subsubsection{Accuracy rule}

For each candidate set-up, the bending errors can be represented in a tolerance tree [1]. The remaining tolerance zone of a toleranced dimension is called slack value. A small slack value for a given toleranced dimension indicates that the accuracy may be a problem. A set-up is not feasible if any slack value is negative. An accuracy score for a candidate set-up can be obtained by multiplying the average of all of the slack values with the average of the two smallest.

\section{Fuzzy set-based evaluation}

In the present sheet metal set-up planning system, handling rules are used to establish the relationships between a bend that has been currently unfolded with the remaining bends that are being considered for unfolding, concerning the handling criteria. The selection of a bend from the remaining bends will satisfy a particular criterion to some extent. Hence, the degrees of satisfaction of the criteria are evaluated with respect to each criterion, for each of the remaining bends. These relationships are represented as fuzzy relations.

\subsection{Problem modelling via fuzzy sets, fuzzy relations and fuzzy matrix}

Let $\boldsymbol{B}=\left\{b_{i} \mid i=1,2, \ldots, a\right\}$ represent the set consisting of all the remaining bends that are being considered for unfolding, where $b_{i}$ is one of the bends.

Let $\boldsymbol{R}=\left\{r_{j} \mid j=1,2,3,4,5\right\}$ represent the set of five criteria in the handling rules, where $r_{j}$ represents one of the criteria.

A fuzzy relation is a mapping from $\boldsymbol{B} \times \boldsymbol{R}$ into $[0,1]$, such that $\mu_{i j}=f\left(b_{i}, r_{j}\right)$ for all pairs $\left(b_{i}, r_{j}\right)$. Since the related sets $B$ and $R$ are finite, a fuzzy relation $f$ on $\boldsymbol{B} \times \boldsymbol{R}$ can be represented as a fuzzy matrix [S], the entries of which are $\mu_{i j}$. Determination of the membership grades $\mu_{i j}$ which may vary anywhere between zero and unity, is based on the rules and fuzzy membership functions presented in the previous sections. The value of unity is assigned to $\mu_{i j}$ when $b_{i}$ satisfies the rule totally, whilst zero is assigned to $\mu_{i j}$ when the particular bend fails the criterion. For example, when there are five remaining bends, the fuzzy relations between these bends and the bend that has been currently unfolded based on the five criteria can be represented as in Table 1.

Let $H C=\left\{b_{k} \mid k=1,2, \ldots, b\right\}$ represent a fuzzy set consisting of bends that would be preforred for next unfolding based on the five handling criteria, where $b_{k}$ is one of the bends. This set can be represented as Eq. (1). The membership grades $h c_{k}$ of the bends in this set represent how well each bend satisfies the five handling criteria, taking into account the relative importance of these criteria.

$H C=\left\{b_{1} / h c_{1}, b_{2} / h c_{2}, \ldots, b_{b} / h c_{b}\right\}$

Let $A P C=\left\{b_{m} \mid m=1,2, \ldots, c\right\}$ represent a fuzzy set consisting of set-ups that would be preferred for next unfolding based on the two accuracy and positioning criteria, where $b_{m}$ is one of the set-ups. The membership values $a p c_{m}$ of the set-ups in this set indicate how well the accuracy and positioning criteria would be satisfied if the particular set-up is selected for performing the next bend to be unfolded. Eq. (2) shows this fuzzy set.

$\boldsymbol{A P C}=\left\{b_{1} / a p c_{1}, b_{2} / a p c_{2}, \ldots, b_{c} / a p c_{c}\right\}$

\subsection{Determination of $\mathrm{HC}$ set}

$H C$ is a fuzzy set consisting of bends that are being considered for next unfolding. The membership grades of the bends in this set indicate how well the criteria of handling are satisfied when these bends are selected. A higher membership value means that the particular bend is preferred to be unfolded next, as compared to a bend with a lower membership value. The relative importance of the handling rules and criteria differs. A hierarchy of these rules has been proposed by Klaasen [15], and tested on eleven industrial components with varying complexity. The following hierarchy (in order of descending importance) has been found to give fair results: (i) compound bend rule; (ii) parallel bend rule; (iii) shape-determining bend rule; (iv) single face rule; 
and (v) combined rotation rule. The relative importance of the rules and criteria is represented as a fuzzy set $\boldsymbol{W}$, as shown in Eq. (3). The following membership values of the criteria have been adopted based on the hierarchy proposed by Klaassen [15].

$$
W[R]=\left\{r_{1} / 1.0, r_{2} / 0.8, r_{3} / 0.6, r_{4} / 0.4, r_{5} / 0.2\right\}
$$

The relative importance of these criteria can now be taken into account to select the next bend. This is achieved by multiplying the respective column vector of this $W$ set with the fuzzy matrix [S] to give a column vector containing the membership values of the bends in the $\boldsymbol{H C}$ set. Therefore, the multiplication procedure can be represented as Eqs. (4) and (j). From Eq. (5), the best bend to unfold next can be found. The next section describes how the best set-up for that bend can be found.

$$
H C(B)=[\mathbf{S}] W(R)
$$

$$
\left[\begin{array}{c}
H C\left(b_{1}\right) \\
H C\left(b_{2}\right) \\
\vdots \\
\vdots \\
H C\left(b_{b}\right)
\end{array}\right]=
$$

$$
\left[\begin{array}{ccccc}
\mu(1,1) & \mu(1,2) & \mu(1,3) & \mu(1,4) & \mu(1,5) \\
\mu(2,1) & \ddots & & & \mu(2,5) \\
\vdots & & \ddots & & \vdots \\
\vdots & & & \ddots & \vdots \\
\mu(b, 1) & \cdots & \cdots & \cdots & \mu(b, 5)
\end{array}\right]\left[\begin{array}{c}
W\left(b_{1}\right) \\
W\left(b_{2}\right) \\
W\left(b_{3}\right) \\
W\left(b_{4}\right) \\
W\left(b_{5}\right)
\end{array}\right]
$$

\subsection{Determination of $A P C$ set}

The accuracy and the 'main part towards operator' rules are evaluated for every possible set-up that could be formed for the next bend. The effect of these two rules can be expressed as:

$a p c_{m}=S V \times M$

where $S V$ is the accuracy score on the basis of the slack values, and $M$ is determined using the membership function in Fig. 5.

A membership value for every bend in the $A P C$ set is calculated using Eq. (6). If the slack values are large, the relative difference will be small, and the main part towards operator rule dictates the preferred set-ups. Slack values indicate how much of the tolerance zone is still available to accommodate errors expected in other set-ups. Therefore, a small slack value indicates problems with respect to accuracy, and a negative slack value indicates that the candidate solution is not feasible [1].

\subsection{Overall procedure}

Since it is almost impossible to consider a complete bending sequence, the part is unfolded one bend at a time. As mentioned earlier, the reverse bending method is adopted in this study. The procedure is described briefly as follows:

[i] Determine the remaining bends to be unfolded.

[ii] Find restrictions, i.e., detect (sub)sequences that will result in collisions.

[iii] Apply handling rules to determine the degrees of satisfaction of the criteria with the selection of each remaining bend.

[iv] Determine the fuzzy matrix, and evaluate to form the various fuzzy sets.

[v] Find and select the best bend to unfold next.

[vi] Determine candidate set-ups for the bend including slack values; select the best set-up.

[vii] Check for accuracy and collisions; if the result of the check is not satisfactory, try other set-ups for the same bend; if no set-up is successful, try another bend.

[viii] Determine if the new product state is not the flat pattern; if no, go to step [i]; otherwise the bend and set-up sequencing is completed.

\section{Example}

In this section, an example is used to illustrate the fuzzy set-based methodology. Fig. 6 shows the example with its tolerance scheme. Views of the bent part are at the top and middle, and the flat pattern is at the bottom of Fig. 6. The tolerance on $F$ is not a problem - this follows immediately from the estimated accuracy of the bend angles. The tolerance on $\mathbf{G}$ does not depend on any bending set-up. Tolerances on $H$ are defined by standards for sheet metal.

In this example, bends 1 and 6 have a fuzzy relation with $\mu=1.0$ from the compound bend rule. Using the fuzzy-set based set-up planning methodology, bend 2 is periormed before bend 3 , and bend 5 is performed before 4 , according to the single face rule, and the shape determining bend rule. However, in addition to the fuzzy set based set-up planning, the present system also performs collision check and detection, together with set-up positioning for the bends sequence that is generated. In this example, the system detected that this bend sequence is not feasible as no set-up can be formed for performing the bends, because there is no suitable positioning feature for bend 3 when bend 2 has been performed first. The same holds for bends 4 and 5 . This is an advantage of using the reverse bending sequence: set-up problems and collision danger are easier to detect. In this example, the system suggested 
Tolerance scheme:

$\begin{array}{ll}\text { A } & 165 \pm 2.0 \\ \text { B } & 40 \pm 0.6 \\ \text { C } & 120 \pm 0.6 \\ \text { D } & 100 \pm 1.0 \\ \text { E } & 23 \pm 0.6 \\ \text { F } & 45^{\circ} \pm 1^{\circ} \\ \text { G } & 20 \pm 0.5 \\ \text { H } & 0.8\end{array}$
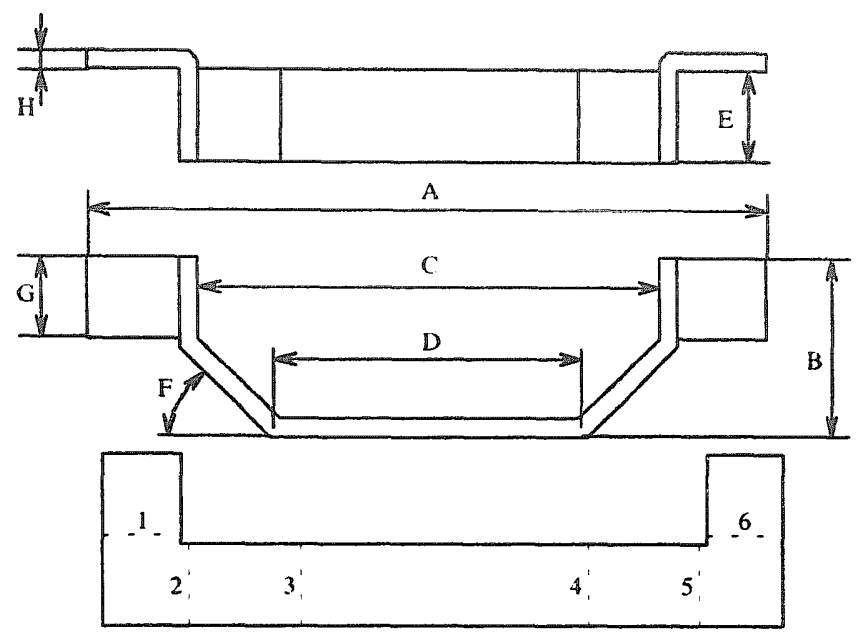

Fig. 6. An example part with its tolerance scheme. that bends 2 and 3, as well as bends 4 and 5 , are to be bent directly after each other, using the parallel bend rule. Fig. 7 shows the set-up plan for this example part. The bend sequence is as follows: bend 1 together with bend 6 , bend 3 , bend 2 , bend 4 , and bend 5 , requiring 5 set-ups. An alternative bends sequence with the same number of set-ups is generated as follows: bend 1 together with bend 6 , bend 4 , bend 5 , bend 3 , and bend 2 .

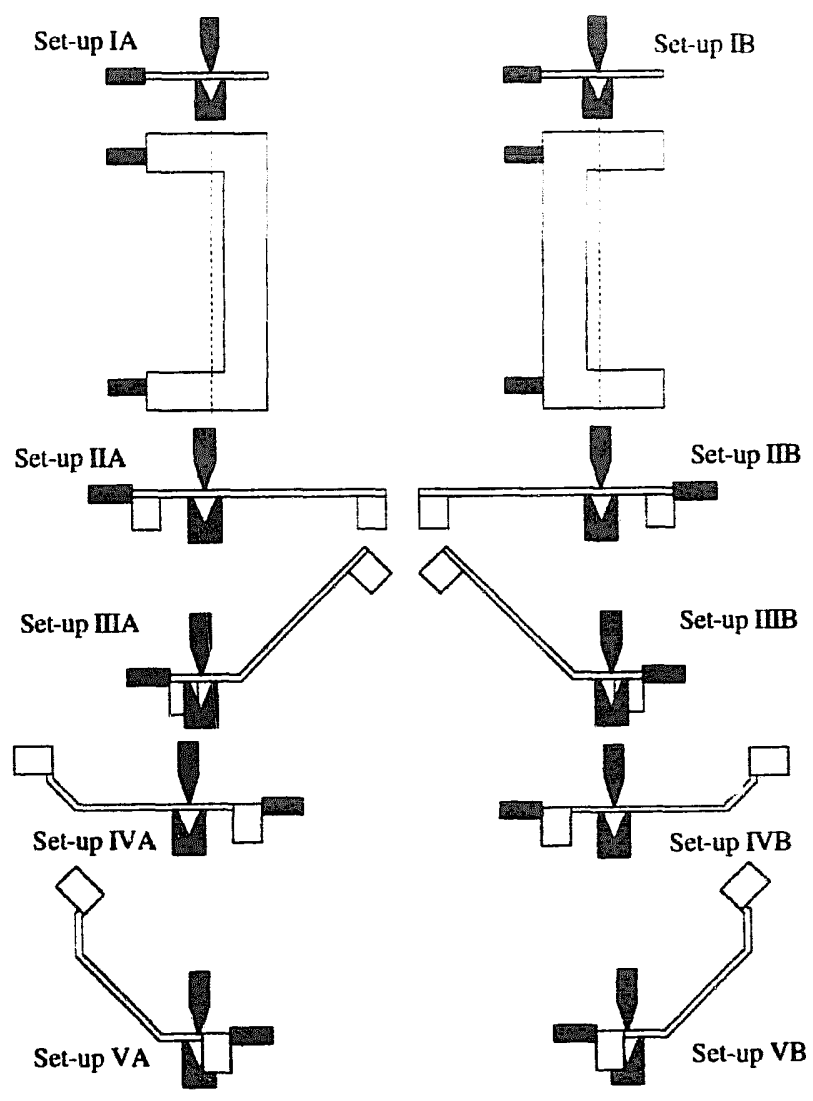

Fig. 7. The set-up and bend sequence.
The system will generate only one of the two bending sequences mentioned above. In the search for the best bend to unfold first, bends 2 and 5 are found to have an equal score. It is arbitrary which bend is selected (either bend may be on top of the list of feasible bends). In the example shown in Fig. 7 , bend 5 was selected. This results in the selection of bend 4 as the next bend to unfold. This selection is mainly driven by the Parallel Bend rule and the Combined Rotation rule. As the next bend to unfold, bend 3 is selected initially but the corresponding set-up does not satisfy the Main Part Towards Operator rule. Therefore, bend 2 is selected and it is processed in the set-up shown in Fig. 7, at the top. Bend 3 is selected subsequently. For the compound bend $(1+6)$ two candidate set-ups are possible. The set-up indicated with 'IB' in Fig. 7 will be selected on the basis of the Main Part Towards Operator rule, unless this set-up is not feasible with respect to accuracy.

\section{Conclusions}

A fuzzy-set based bend and set-up sequencing methodology for sheet metal working has been presented. By modelling the various handling, accuracy and positioning rules for sequencing bends and set-ups, the system allows the relative importance of the handling and accuracy issues to be incorporated into the set-up sequencing procedures. The highly experienceand heuristic-based conventional set-up planning procedures in sheet metal bending are aptly modeled in this computer-automated bends and set-up sequencing system, using the fuzzy set theory. In addition, the capability of the system to perform collision checks and detection, and set-up positioning, simultaneously with set-up planning ensures that the set-up plans that are generated are feasible. 


\section{References}

[1] L.J. De Vin, Computer aided process planning for the bending of sheet metal components, PhD Thesis, University of Twente, Enschede, NL, 1994.

[2] F. Wang, L. Chang, Determination of the bending sequence in progressive die design, Proc. IMech Part B [Special issue]. J. Eng. Manuf. 209 (B1) (1995) 67-73.

[3] L.A. Zadeh, Fuzzy sets, Information and Control, 8, 339-353.

[4] Z.-C. Lin, G.-J. Peing, An investigation of an expert system for sheet-metal bending design, J. Mater. Process. Technol. 43 (2-4) (1994) 165-176.

[5] S.K. Ong, A.Y.C. Nee, Application of fuzzy set theory to set-up planning, Ann. CIRP 43 (1) (1994) 137-143.

[6] K. Osakada, G. Yang, K. Mori, Determination of optimum forming path in three-roll bending by combination of fuzzy reasoning and finite element simulation, Ann. CIRP 42 (1) (1993) 291-294.

[7] G. Yut, T.C. Chang, A study of automated process plannin for sheet metal products, in: Proc. 1993 NSF Design \& Manu- facturing Systems Conference, SME, Vol. 2, 1993, pp. 12991305.

[8] C. Baker, Developments in press brakes and folders, Sheet Met. Ind. 67 (4) (1990) 203.

[9] M. Hoffmann, U. Geißler, M. Geiger, Computer-aided generation of bending sequence for die-bending machines, J. Mater. Process. Technol. 30 (1) (1992) 1-12.

[10] P. Vidlicka, Computer aided manufacture of sheet metal components, Sheet Met. Ind. 70 (6) (1993) 22-25.

[11] R. Ehrismann, J. Reissner, Intelligent manufacture of laser cutting, punching and bending of parts, J. Robotics \& ComputerIntegrated Manuf. 4 (3/4) (1988) 511-515.

[12] K.H. See Toh, H.T. Loh, A.Y.C. Nee, K.S. Lee, A feature-based flat pattern development system for sheet metal parts, J. Mater. Process. Technol. 48 (1995) 89-95.

[13] J. Fleischer, Automatische ermittlung der abkantreihenfolge, VDI-Z 31 (5) (1989) 44-46.

[14] M. Geiger, M. Hoffmann, B. Kluge, Inferenzmaschine für ein Biegestadienplanungssystem, ZwF 87 (5) (1987) $261-264$.

[15] E. Klaassen, Bending sequence pre-determination and collision checking for PART-S, MSc Thesis, University of Twente, 1993. 